\title{
Cell-free Protein Expression of Membrane Proteins Using Nanolipoprotein Particles
}

\author{
Federico Katzen, Julia E. Fletcher, Jian-Ping Yang, Sanjay Vasu, Todd Peterson, and Wieslaw Kudlicki \\ Invitrogen Corporation, 5791 Van Allen Way, Carlsbad, CA 92008
}

\section{INTRODUCTION}

The paucity of membrane protein science reflects the hurdles that these proteins impose on their expression and purification. Expression of membrane proteins in vivo frequently results in cell toxicity, protein aggregation, misfolding, and low yield. Vesicle preparation, detergent extraction, and membrane protein reconstitution into liposomes are processes that inexorably lead to constraints on protein accessibility, surface immobilization, and activity assays. We report a cell-free protein expression approach for expressing and inserting integral membrane proteins into water soluble particles (brand name MembraneMax' ${ }^{\mathrm{TM}}$, offered tag-free or histidine tagged) composed of a phospholipid membrane bilayer surrounded by a scaffold protein. The method circumvents the need of extracting and reconstituting the product into membrane vesicles. Moreover, the planar nature of the membrane support makes the protein freely accessible from both sides of the lipid bilayer.

\section{MATERIALS AND METHODS}

Materials and methods are described in the Journal of Proteome Research (http://pubs.acs.org/cgi-bin/abstract. cgi/jprobs/asap/abs/pr800265f.html).

\section{RESULTS AND DISCUSSION}

A diagram of the method is shown in Figure $1 \mathrm{~A}$. Proof of concept was demonstrated using bacteriorhodopsin, a 7 transmembrane segment protein derived from Halobacterium salinarum, which was co-purified with histidine tagged particles (MembraneMax ${ }^{\mathrm{TM}} H N$, Figure 1B). When the particles were included in the reaction, the protein folded in its native conformation, as it became fully water soluble and exhibited its characteristic purple color (Figure 1C).

The approach was further validated by assessing the solubility of a host of membrane proteins of different topologies, sizes, sources, and proposed roles. Without exception, the proteins significantly increased their solubility in the presence of the MembraneMax reagent (Figure 1).
A

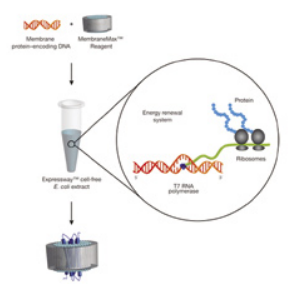

B

C
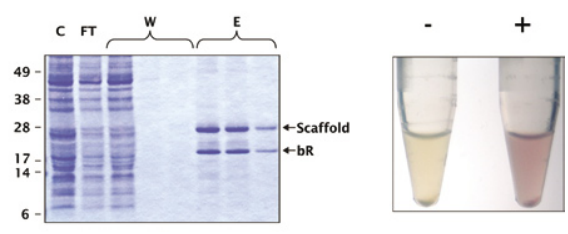

Figure 1. Complex formation between MembraneMax ${ }^{\mathrm{TM}} H N$ and bacteriorhodopsin. A) Cell-free expression and solubilization of membrane protein targets. B) Non-tagged bacteriorhodopsin was expressed using the Expressway ${ }^{\mathrm{TM}}$ cell-free protein expression reaction kit in the presence of MembraneMax TM $H N$. Proteins were purified over a $\mathrm{Ni}^{2+}$ column and separated by SDS electrophoresis. C) Photograph of the cell-free protein expression reaction tubes where bR was expressed in the presence (+) or absence (-) of MembraneMax ${ }^{\mathrm{TM}} H N$ (all-trans retinal was included in both reaction vessels). C, crude extract; $F$, flow through fraction; W, wash fractions; $E$, imidazole eluted fractions.

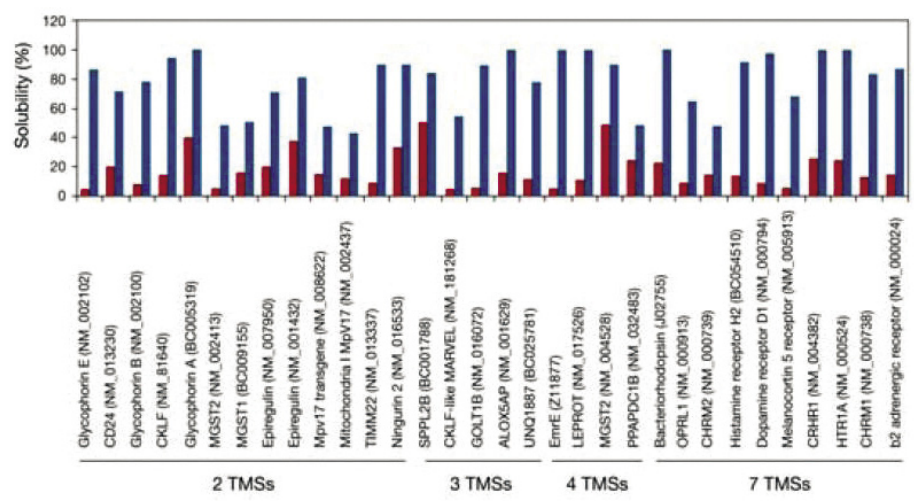

Figure 2. Soluble membrane protein expression achieved using the MembraneMax ${ }^{\mathrm{TM}} H N$ kit. Proteins were expressed in the presence (blue) or absence (red) of MembraneMax ${ }^{\mathrm{TM}} \mathrm{HN}$ reagent. For the analyzed data set, the overall solubility increased from $17.3 \pm 2.2 \%$ (in the absence of MembraneMax ${ }^{\mathrm{TM}} H M$ ) to $78.8 \pm 3.4 \%$ (in the presence of MembraneMax ${ }^{\mathrm{TM}} H M$ ). GPCRs exhibited a remarkable increase in solubility in the presence of NLPS. TMS $=$ transmembrane segments.

Our method for the expression of integral membrane proteins is unlike conventional membrane protein methods in that: (i) it delivers high product yield ( $\mathrm{ng}$ to $\mathrm{mg} / \mathrm{ml} \mathrm{reac-}$ tion) in under 3 hours, (ii) it renders soluble (dispersed) MPs without extraction/reconstitution steps, (iii) it is high throughput-amenable, (iv) it provides purification flexibility when expressing native or tagged membrane proteins, (v) it is compatible with a variety of cell-free lysates, vi) produces a more homogeneous sample of membrane proteins than the liposome/vesicle approach, and (vii) the final complex is freely accessible from both sides of the lipid bilayer. 